This is a post-peer-review, pre-copyedit version of an article published in Advances in Experimental Medicine and Biology.

\title{
Application of Photogrammetry in Biomedical Science
}

Ronja Struck, Sara Cordoni, Sofia Aliotta, Laura Pérez-Pachón and Flora Gröning

Flora Gröning (corresponding author)

School of Medicine, Medical Sciences and Nutrition, University of Aberdeen, Aberdeen, United Kingdom

E-mail: f.groening@abdn.ac.uk

Keywords: digital 3D reconstruction, multi-camera setup, structure-from-motion stereophotogrammetry, microphotogrammetry, medicine

\begin{abstract}
Photogrammetry is an upcoming technology in biomedical science as it provides a non-invasive and cost-effective alternative to established 3D imaging techniques such as computed tomography. This review introduces the photogrammetry approaches currently used for digital 3D reconstruction in biomedical science and discusses their suitability for different applications. It aims to offer the reader a better understanding of photogrammetry as a $3 \mathrm{D}$ reconstruction technique and to provide some guidance on how to choose the appropriate photogrammetry approach for their research area (including single- versus multi-camera setups, structure-from-motion versus conventional photogrammetry and macro- versus microphotogrammetry) as well as guidance on how to obtain high-quality data. This review highlights some key advantages of photogrammetry for a variety of applications in biomedical science, but it also discusses the limitations of this technique and the importance of taking steps to obtain high-quality images for accurate 3D reconstruction.
\end{abstract}




\section{Introduction}

Digital 3D reconstruction has become a vital tool in the study of the structure and functions of the human body and biomedical scientists can now choose from a range of well-established imaging techniques for 3D modelling, including computed tomography (CT) and magnetic resonance imaging (MRI) as well as laser-scanning microscopy. Photogrammetry is a less common approach for digital 3D reconstruction compared to these techniques, but due to its ease of use and costeffectiveness, it is an interesting alternative for biomedical scientists.

The word photogrammetry is a combination of three Greek root words: "phot", "gramma" and "metrein", respectively meaning "light", "something drawn" and "measure". Collectively, these terms translate to "measuring graphically by means of light" (Ey-Chmielewska et al. 2015).

Photogrammetry is broadly defined by the American Society of Photogrammetry and Remote Sensing (ASPRS) as the science of obtaining precise information about the surface structure of an object or a particular environment by a recording device which is not in direct contact with the object that is being studied (Estes et al. 2001). This means that photogrammetry can be applied in every circumstance where the object of interest can be photographically documented (Luhmann et al. 2006).

Photogrammetry was first used in Medicine by American physician Holmes in 1863 to study the gait of civil war amputees in an effort to design prosthetics to aid rehabilitation (Lane 1983). However, recent advances in computer software development now allow us to use overlapping images to create detailed 3D surface models of biological structures, a procedure known as stereophotogrammetry or close-range photogrammetry (Villa 2017).

This chapter will provide an overview of the photogrammetry approaches currently used for digital 3D reconstruction in biomedical science and discuss their suitability for different applications. We aim to offer the reader a better understanding of this upcoming technology and to provide some guidance on how to choose the appropriate photogrammetric approach for their research area and to obtain highquality data. 
We have used PubMed and Science Direct as main online databases for this literature research, with key words including "photogrammetry", "microphotogrammetry", "biomedical science", "stereophotogrammetry", "photogrammetry review", "structure-from-motion photogrammetry", "photogrammetry review", "photogrammetry + prosthetic", "multi-camera photogrammetry", "sfm vs laser scanning", "photogrammetry + tumor", "stereoscopic camera types" and "stereoscopy+dermatology". We included studies on humans as well as animal studies.

\section{Single-Camera Setups}

To create a 3D model from photographs, images have to be taken from multiple angles. This is achieved by either moving one camera around the object (singlecamera setup) or arranging multiple cameras around the object (multi-camera setup). Although the 3D reconstructions based on single and multi-camera setups are similarly accurate (Liu et al. 2015; Villa 2017), there are some basic differences in the characteristics of the approaches (Table 1).

Table 1. Overview of the main characteristic of single-versus multi-camera setups. $\mathrm{IOP}=$ internal orientation parameters, EOP = external orientation parameters

\begin{tabular}{|c|c|c|c|}
\hline & \multicolumn{2}{|c|}{ Single-Camera Setup } & \multirow{2}{*}{$\begin{array}{c}\text { Multi-Camera } \\
\text { Setup }\end{array}$} \\
\hline & $\begin{array}{c}\text { Non-Stereoscopic } \\
\text { Camera Setup }\end{array}$ & $\begin{array}{l}\text { Stereoscopic } \\
\text { camera setup }\end{array}$ & \\
\hline Number of cameras & 1 & Usually 2 & $>1$ \\
\hline $\begin{array}{l}\text { Number of pictures } \\
\text { taken per shot }\end{array}$ & 1 & 1 & $\begin{array}{c}\text { equal to the number } \\
\text { of cameras }\end{array}$ \\
\hline Resulting 3D model & $\begin{array}{l}\text { Complete 3D } \\
\text { reconstruction } \\
\text { possible }\end{array}$ & $\begin{array}{l}\text { Partial } \\
\text { reconstruction only }\end{array}$ & $\begin{array}{l}\text { Complete 3D } \\
\text { reconstruction } \\
\text { possible }\end{array}$ \\
\hline $\begin{array}{l}\text { Operation } \\
\text { complexity }\end{array}$ & Simple & Medium & Complex \\
\hline $\begin{array}{l}\text { Time required for } \\
\text { setup }\end{array}$ & Short & Simple & Long \\
\hline $\begin{array}{l}\text { Image acquisition } \\
\text { time }\end{array}$ & Long & Medium & Short \\
\hline $\begin{array}{l}\text { IOP and EOP } \\
\text { consistency }\end{array}$ & $\begin{array}{l}\text { IOP (e.g. zoom or } \\
\text { focus) instabilities }\end{array}$ & Short & $\begin{array}{l}\text { IOP and mounting } \\
\text { instabilities }\end{array}$ \\
\hline Costs & Low & Medium & High \\
\hline
\end{tabular}


A single-camera setup uses only one camera and is consequently cheaper and easier to setup than a multi-camera setup. These advantages might be the reasons why it is used more frequently in biomedical sciences than the multi-camera setup. There is a wide range of biomedical applications of single-camera setups, ranging from the documentation and measurement of scars and lesions (Stekelenburg et al. 2015; Stekelenburg et al. 2013; Villa 2017) over the assessment of lung volumes (Ripka, Ulbricht and Gewehr 2014) to electrode localisation for electroencephalography (EEG) (Qian and Sheng 2011).

A common type of single-camera setup is the use of a stereoscopic camera. This is a single camera that takes two pictures from different angles in one shot. As only two images are used for the 3D reconstruction, this approach yields partial 3D reconstruction rather than a 360-degree reconstruction of the object of interest.

There are different methods to achieve the simultaneous acquisition of two images in a stereoscopic camera: e.g. using a multi-lens camera or lens splitter (Ueno et al. 1989; Stekelenburg et al. 2013). However, the most commonly used stereoscopic camera in biomedical sciences is a modified single lens camera with a lens splitter (Stekelenburg et al. 2013).

Lens splitters are devices that split the lens in such a way that two images from different viewpoints can be taken in a single shot (Stekelenburg et al. 2013). They usually come with a dual light pointer system and software that creates a 3D model (Stekelenburg et al. 2015). The dual light pointer system consists of two angled light pointers. Their beams converge at the exact distance that the picture should be taken at (Stekelenburg et al. 2015). At this distance, the reconstruction from the two images acquired is the most successful as the accompanying software is designed to merge images taken from known relative 3D locations. Taking the pictures at the recommended distance assures that the 3D locations the pictures are taken at and those the software matches pictures from are the same.

There are different sizes of stereoscopic cameras available for different biomedical applications (e.g. Quantificare: 3D LifeViz® Micro for wrinkle or scar visualisation or 3D LifeViz ${ }^{\circledR}$ Infinity for maxillofacial or breast surgery). In addition, suppliers offer 
supplementary software that simulates the effects of potential treatment options (e.g. Quantificare). For a patient to be able to visualise their appearance after surgery is an important tool in making the decision for a specific treatment. This can be especially useful in cosmetic or reconstructive surgery (Gibelli et al. 2018).

A common issue using a single-camera setup are differing internal orientation parameters (IOPs) such as zoom or focus. Using a stereoscopic camera, an approach relatively common in maxillofacial surgery and skin assessment (Stekelenburg et al. 2013; Stekelenburg et al. 2015), eliminates this problem, as all pictures for a single reconstruction are taken at the same time and with the same camera. Therefore, the IOPs do not vary between the images.

Stereoscopic cameras that take only two pictures for a 3D reconstruction are particularly suitable for applications that do not require 360-degree information (e.g. in craniofacial surgery (Stekelenburg et al. 2013) when a reconstruction of the back of the head is not required). Compared to non-stereoscopic single camera or multicamera setups, stereoscopic cameras are easy to use, do not require a lot of space and provide fast results (Stekelenburg et al. 2013) that can be discussed with the patient immediately.

\section{Multi-Camera Setups}

A multi-camera setup uses multiple cameras commonly mounted on a metal frame or scaffold. The additional cameras do not only make this setup more expensive, but also more complex in the setup and initial calibration, as not only IOPs of the cameras have to be matched, but also the exterior orientation parameters (EOPs, such as the position and orientation of the camera) must be considered. EOPs are concerned with the orientation of the cameras in relation to their mounting system (Habib et al. 2014). These difficulties cause a multi-camera setup to only be preferable when the object is either moving or many objects can be digitised in one setup.

Multi-camera setups are a the most time-efficient solution for 3D reconstruction, however, if many objects of similar shape and size are to be digitised, as only one setup is required with little further calibration is after the initial installation. For 
example, Leipner and colleagues (2016) designed a chamber with 64 cameras for the image acquisition of persons in standing posture, a setup designed for victim and suspect documentation in forensics. With all objects being of similar appearance a multi-camera setup is the preferable option for this application. The object should, however, be large enough for multiple cameras to be positioned around it at a reasonable distance.

Multi-camera setups are also convenient when the photographed object moves and therefore photo acquisition must occur quickly. For example, Zemčík and colleagues (2012) used this approach to investigate the effect of manual perineal protection during birth. In this case, the object, i.e. the perineum, moved during the period of image acquisition. Therefore, a single-camera setup would have been insufficient, as it does not allow to capture enough angles of the object in the same state (Zemčík et al. 2012).

\section{Structure-from-Motion Photogrammetry versus Conventional Photogrammetry}

To create a 3D model based on photogrammetry, images have to be aligned using points that are shared between the images. Two main approaches can be used for this point identification process: conventional and structure-from-motion photogrammetry (SFM) (Figure 1, see Table 2 for an overview of the requirements and outputs of these two approaches). 
Figure 1. Approaches used in stereophotogrammetry and differences in the image alignment process between the approaches

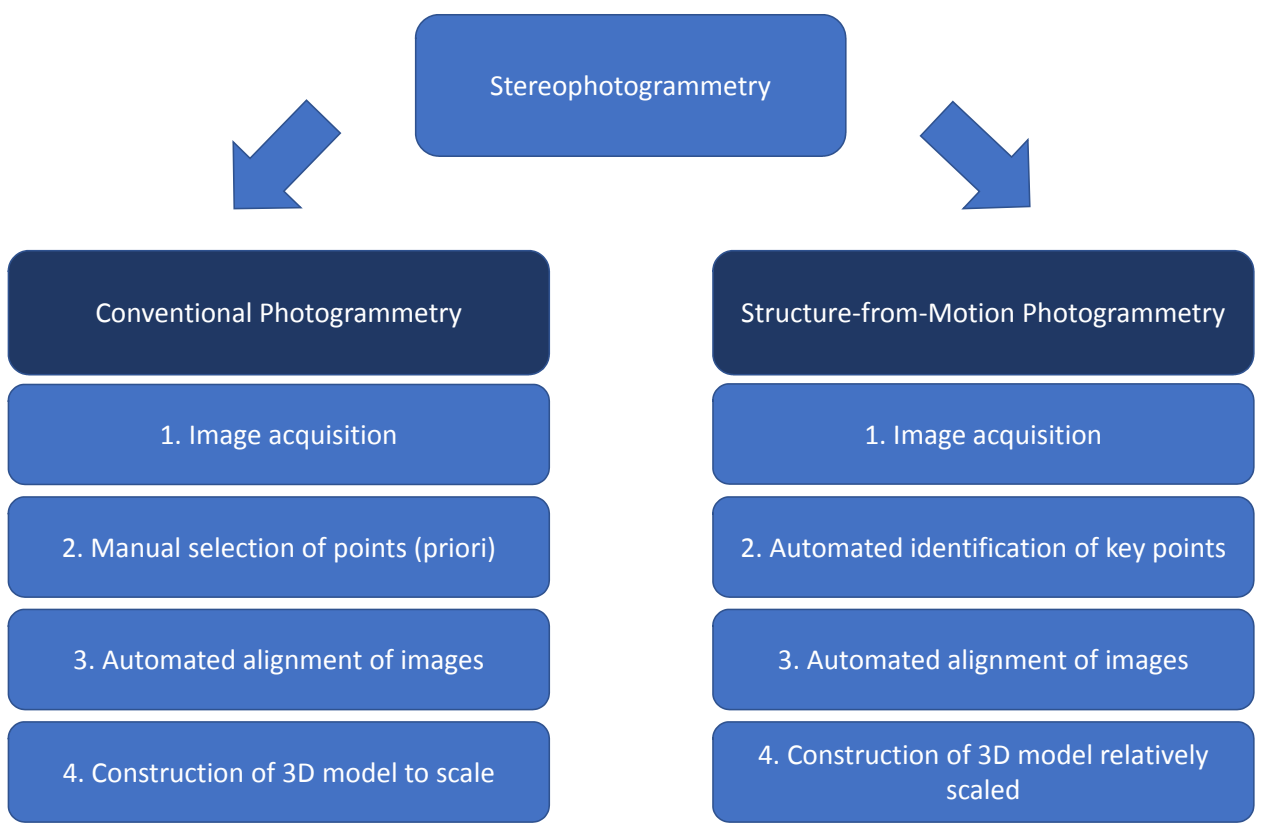


Table 2. Structure-from-motion versus conventional photogrammetry

\begin{tabular}{|c|c|c|}
\hline & $\begin{array}{l}\text { Structure-from-Motion } \\
\text { Photogrammetry }\end{array}$ & $\begin{array}{l}\text { Conventional } \\
\text { Photogrammetry }\end{array}$ \\
\hline $\begin{array}{l}\text { Creation of point cloud } \\
\text { from images }\end{array}$ & $\begin{array}{l}\text { Automated process } \\
\text { using key points to } \\
\text { match large sets of } \\
\text { images with overlapping } \\
\text { areas or from a video }\end{array}$ & $\begin{array}{l}\text { Uses priori (network } \\
\text { of targets with } \\
\text { known 3D locations) }\end{array}$ \\
\hline Requirements & $\begin{array}{c}\text { At least } 60 \% \text { of overlap } \\
\text { between adjacent } \\
\text { pictures }\end{array}$ & $\begin{array}{c}\text { Priori, network of } \\
\text { points with known } \\
\text { 3D locations } \\
\text { or } \\
\text { 3D location and } \\
\text { pose of the } \\
\text { camera(s) }\end{array}$ \\
\hline Scale of output & $\begin{array}{c}\text { Arbitrarily scaled } \\
\text { coordinate system } \\
\text { (scaling can be done in a } \\
\text { separate step) }\end{array}$ & $\begin{array}{l}\text { Scaled coordinate } \\
\text { system because the } \\
\text { priori relate it to the } \\
\text { dimensions of the } \\
\text { real world }\end{array}$ \\
\hline
\end{tabular}

In conventional photogrammetry, the distance between camera and object must be known as it is used to calculate the scale and match the pictures. For this, the software uses either known 3D locations (priori) of a network of points on the pictures or known 3D locations and orientations of the camera(s), both of which need to be manually identified (Westoby et al. 2012). In SFM on the other hand algorithms detect and match key points in overlapping areas of the pictures automatically (Skarlatos and Kiparissi 2012).

SFM is a fairly new approach of photogrammetry, as it relies on algorithms which have recently been advanced by significantly improving their accuracy (Skarlatos and Kiparissi 2012). In this type of photogrammetry, the 3D reconstruction of the object is performed using automated matching processes (Westoby et al. 2012). Opposed to conventional photogrammetry, SFM uses algorithms to detect and 
match key points in overlapping areas of the pictures automatically (Skarlatos and Kiparissi 2012). These key points are then arranged in a 3D point cloud (Marčiš 2013).

The reconstruction is based on information acquired either from video images or photographs taken from several angles around the object. The resulting model is created in an unscaled coordinate system, because the 3D locations extracted from the images are relative to each other rather than to scale (Villa 2017).

Leipner and colleagues (2016) and Qian and Sheng (2011) used SFM in their studies on the reconstruction of living persons in a standing position and using EEG electrode localisation. Despite some limitations depending on the specific application, the authors of both studies highlight the potential of this approach in biomedical sciences.

SFM is more user-friendly than conventional photogrammetry given the fact that the images matching process is automated. This may be the reason why, based on our literature review, SFM appears to be the preferred approach in biomedical sciences. Conventional photogrammetry is most commonly applied through camera setups such as stereoscopic cameras (e.g. Stekelenburg et al. 2013; Stekelenburg et al. 2015) with accompanying software solving the scene by considering the parameters of e.g. the lens splitter or multiple lenses, by which the camera operates.

\section{Microphotogrammetry}

Microphotogrammetry is a 3D reconstruction tool that uses scanning electron microscopy (SEM) instead of a standard photographic camera for image acquisition. Thus it achieves much higher resolutions (i.e. nanoscale resolution and depending on the instrument less than one nanometer) compared to other approaches of photogrammetry that use macroscopic image acquisition. Microphotogrammetry has allowed 3D reconstruction through SEM since the 1970s (Ball et al. 2017; Tafti et al. 2016).

In SEM a focused beam of electrons interacts with the atoms on the surface of a sample. This produces signals that are picked up by a detector and then converted 
into a 2D image of the surface topography of the sample (Ball et al. 2017). However, the lack of 3D data can limit the interpretation of the images and their quantitative analysis. Microphotogrammetry provides an approach to create 3D models of the surface topography based on these 2D images. In general, 3D reconstruction from 2D micrographs is achieved through the same basic procedure as in macroscopic stereophotogrammetry where a point cloud, a mesh and ultimately a textured 3D model is produced (Tafti et al. 2016).

Surface image acquisition and subsequent reconstruction techniques using SEM can be categorised into three main groups: 1) single-view, 2) multi-view and 3) hybrid, which is a combination of the first two. In single-view techniques, a range of electron beam trajectories captures images from a single perspective while in multi-view approaches a combination of viewpoints is used to create a 3D model. Hybrid approaches combine the advantages from both single- and multi-view approaches, but they are yet to be fully designed and validated (Tafti et al. 2015). Single-view setups are a well-studied and the most adopted approaches in the literature (Baghaie et al. 2017).

The study from Ball et al. (2017) is a prime example where microphotogrammetry has been applied within the field of bioscience by using it to study microinvertebrates. The authors managed to create a high-resolution 3D model of an insect's head up to $1000 x$ magnification using single-view techniques.

By contrast, Eulitz and Reiss (2015) moved away from the traditional method of 3D reconstruction using single-view methods and proposed a multi-view approach that adopts the fundamental characteristics of optical stereophotogrammetry. As in optical stereophotogrammetry, they created 3D models from series of overlapping images of the sample, in this case a rabbit kidney glomerulus. These images were produced by rotating the sample under the fixed detector to acquire data from multiple angles, a variant of multi-view techniques (Baghaie et al. 2017). The result showed an enhanced 3D reconstruction quality and better preservation of the original specimen (Eulitz and Reiss 2015). 
Microphotogrammetry shows to be a promising high-resolution 3D modelling technique, however not every microscopic object is suitable for 3D reconstruction (Tafti et al. 2016). In addition, the sample has to be small enough to fit into an SEM chamber. As in optical and macroscopic stereophotogrammetry, samples also need to be mounted in a stable way and should not undergo deformations during image acquisition. Moreover, the surface micro-anatomy of an object needs to be easily traceable by the electron beam. This means that rougher surfaces with different superficial patterns as well as bright areas are preferred over flat, smooth and dark surfaces (Tafti et al. 2016). In conventional SEM, vacuum and sputter coating are required (Faith et al. 2006). This is an invasive technique which is only suitable for ex vivo samples. This process obliterates the sample for most other analyses after the SEM scan. Environmental SEM, on the other hand, does not require sputter coating and leaves the sample intact (Griffith and Danilatos 1993), thus allowing the sample to be analysed with other techniques.

\section{Why to choose photogrammetry for digital 3D reconstruction?}

Photogrammetry is becoming a popular technique for a variety of applications due to its portability, non-invasiveness and cost-effectiveness. Alternative methods of 3D modelling, such as 3D laser scanners, computed tomography (CT) or magnetic resonance imaging (MRI) tend to be significantly more expensive and often larger and heavier than photogrammetry equipment (Chandler and Buckley 2016; Evin et al. 2016). Furthermore, photogrammetry does not require extensive training or the attendance of a trained professional (Villa, 2017). Compared to CT, photogrammetry does not use ionising radiation and therefore provides a cheap and low-risk alternative to CTs in, for example, dental or postural assessment (Saad et al. 2012). Photogrammetry can be used to minimise the radiation exposure for patients by using it in combination with conventional spinal curvature assessments and checkups (Liu et al. 2015). As photogrammetry provides textured models, unlike CT- or MRI-based models, it is an attractive option for various dermatological applications (Stekelenburg et al. 2015). The most important advantages of photogrammetry, however, are its ease of use (Evin et al. 2016) and relative inexpensiveness making it accessible to a wide range of users. Many photogrammetry software packages are 
free or affordably priced and some even run on smartphones (Chandler and Buckley 2016).

In addition to these specific advantages, photogrammetry shares the advantages other digital 3D imaging techniques, e.g. concerning data storage and reproducibility of measurements. Photogrammetric datasets are easily storable and can readily be reassessed. This is highly advantageous specifically in fields such as pathology in which the original specimen or sample might be available for a limited time (Villa, 2017). Any measurements taken on 3D photogrammetry models can be aided by software (Stekelenburg et al. 2013). As a result, these measurements tend to be more accurate and show higher intra- and inter-operator reliabilities compared to manual measurements (Villa 2017). Evin and colleagues (2016) compared to the accuracy of photogrammetry models of digitized skulls to those obtained with 3D laser scanning and showed that photogrammetry provides a reliable alternative to conventional 3D laser scanners for surface modelling. However, there are currently limited data available on the accuracy of photogrammetry models compared to other digital 3D reconstruction approaches.

\section{How to obtain high-quality 3D reconstructions with photogrammetry: a brief guide}

The wide range of applications of photogrammetry in biomedical science shows that it is a very versatile approach for virtual 3D reconstruction. However, to make best use of this powerful technique, it is important to plan a new project carefully, be aware of the limitations of the technique and take steps to optimize image acquisition and image processing. The following guidelines are based on published papers as well as the authors' own experiences.

\subsection{Features of the objects to be digitised}

Most suitable objects for photogrammetry are compact without holes, thin protrusions or folded surfaces (e.g. the crotch area of a person). In addition, photogrammetry and especially SFM cope best with grainy textures rather than unicoloured surfaces. 
Photogrammetry software used to generate point clouds has a tendency to correct for missing information by using the surrounding information to automatically fill holes via smoothing (Marčiš, 2013). Thus holes or other shaded areas may be treated as part of the texture by the software which can lead to the creation of artefacts in the model (Marčiš, 2013; Chandler and Buckley, 2016). Another issue is demonstrated by the incomplete reconstruction of thin protrusions (e.g. thin bony processes) due to too little matching information (Probst et al. 2018). The greater curvature on the thin structure compared to a larger one causes every point of it to be seen from fewer angles and therefore in fewer images. If there are not enough overlapping images (i.e. sharing a sufficient number of surface points seen from different angles), the software cannot match the points correctly in the 3D reconstruction which leads to an incomplete reconstruction.

If objects are to be digitized that do not fit the criteria outlined above, other 3D imaging techniques such as CT or MRI scanning can be considered. Those techniques are also more suitable if the internal structure of an object is of interest. Whereas photogrammetry provides solely surface information, those techniques scans show both external as well as internal structures (Villa et al. 2017).

\subsection{Choice of the photogrammetry approach}

The most suitable photogrammetry approach depends on the application and scale of the study. Table 3 provides a brief overview of the main approaches and examples of their applications in biomedical science. It is important to carefully consider the advantages and disadvantages of each approach for a specific study before investing in equipment. 
Table 3. The main photogrammetry approaches and examples of their application in biomedical sciences

\begin{tabular}{|c|c|c|c|}
\hline & \multicolumn{2}{|c|}{ Single-Camera Setup } & \multirow{2}{*}{$\begin{array}{l}\text { Multi-Camera } \\
\text { Setup }\end{array}$} \\
\hline & $\begin{array}{l}\text { Non-Stereoscopic } \\
\text { Camera Setup }\end{array}$ & $\begin{array}{l}\text { Stereoscopic } \\
\text { Camera Setup }\end{array}$ & \\
\hline $\begin{array}{l}\text { Structure-from-Motion } \\
\text { Photogrammetry }\end{array}$ & $\begin{array}{l}\text { Documentation and } \\
\text { measurement of } \\
\text { model and live } \\
\text { human and animal } \\
\text { structures (Gibelli } \\
\text { et al. 2018; Evin et } \\
\text { al. 2016; Qian and } \\
\text { Sheng 2011; } \\
\text { Ritschl et al. 2018; } \\
\text { Villa 2017) } \\
\text { Assessment of lung } \\
\text { volumes (Ripka et } \\
\text { al. 2014) }\end{array}$ & & $\begin{array}{c}\text { Documentation of } \\
\text { body stature and } \\
\text { natural head } \\
\text { position (Leipner et } \\
\text { al. 2016; Liu et al. } \\
\text { 2015) } \\
\text { Measurement of } \\
\text { diseased tissues } \\
\text { (Denise et al. 2013 } \\
\text { O'Meara et al. } \\
\text { 2012) }\end{array}$ \\
\hline $\begin{array}{l}\text { Conventional } \\
\text { Photogrammetry }\end{array}$ & & $\begin{array}{c}\text { Measurement of } \\
\text { skin features } \\
\text { (Stekelenburg et al. } \\
\text { 2013; Stekelenburg } \\
\text { et al. 2015) }\end{array}$ & $\begin{array}{l}\text { Analysis of the } \\
\text { human body in } \\
\text { movement (Zemčík } \\
\text { et al. 2012) }\end{array}$ \\
\hline Microphotogrammetry & $\begin{array}{l}\text { Visualisation of } \\
\text { microscopic animal } \\
\text { structures (Ball et } \\
\text { al. 2017; Eulitz and } \\
\text { Reiss 2015) } \\
\text { Visualisation of } \\
\text { dental } \\
\text { implant (Glon et al. } \\
\text { 2014) }\end{array}$ & & \\
\hline
\end{tabular}

\subsection{Equipment}

Successful 3D models can be created from all types of cameras, ranging from simple phone camera to SLR cameras (Petriceks et al. 2018). However, the limited accuracy of models based on smartphone images must be considered. Hernandez and Lemaire (2016), for example, detected that their smartphone-based models are 
about $2 \mathrm{~mm}$ larger than the real-world object. For maximum accuracy, a professional SLR camera is therefore recommended. In addition, a camera with a fixed focal length lens (e.g. $50 \mathrm{~mm}$ ) is preferable compared to a variable focal length lens. If a variable focal length lens is used, maintaining the same focal length over the course of the shooting is recommended.

Using a tripod is highly recommendable, as it helps to stabilise the camera, ensuring sharp pictures. A remote control is another useful accessory as pressing the shutter button can cause vibration to the camera. Another helpful accessory is a turntable. A turntable is especially important when space around the photographed object is limited; it also helps arranging the tripod and lights in a static location. Usually a reference system is attached to the turntable to help the software with the reconstruction of the photographed object. In addition, a black background (e.g. black cloth) will simplify the masking process and it will afterwards help the software recreating the photographed object without any interferences from the background. When photographing shiny or reflective objects, a circular polarizing filter is advised to reduce the bright spots caused by reflection. An alternative method can be simply covering the photographed object in e.g. patterned tape (Hernandez and Lemaire 2017).

\subsection{Camera Settings}

The key for achieving a detailed and accurate 3D model is high quality images (Skarlatos et al. 2012). Using an appropriate exposure is particularly important. The light meter in the camera usually changes the actual colour of the photographed object into a natural grey. For instance, if there are dark objects (e.g. black background) in the frame, the light meter of the camera will tend to brighten the photograph to make those dark tones look like a neutral grey. If the photographed object has many bright tones (e.g. a hand of a cadaveric specimen with some areas covered in skin, fascia or tendons) the camera will often darken the image. Therefore, the exposure needs to be adjusted manually. A constant exposure usually reduces the work in the post-processing stage. Consequently, artificial light is preferred compared to natural light. Artificial light is constant and adjustable depending on colours and tones of the photographed object. Flash is usually not 
recommended for photogrammetry since it creates a lot of shadows and darkness in the non-illuminated part of the scene (Marčiš et al. 2013).

Shutter speed and aperture should also be taken in consideration. A greater field of depth is provided with a small aperture (higher numbered like f/16) and a shutter speed of at least 1/50th of a second (Villa et al. 2017). Although, they should be checked and adjusted manually depending on the object photographed and the light used.

\subsection{Camera Positioning and Shooting}

The optimal number of photos required depends on the size and complexity of the photographed object. Photographs should be taken from different angles to capture information from the whole surface of the photographed object (Chandler et al. 2016). Preferably, a photo is taken every 10-15 degrees (horizontally and vertically). A 50-60\% overlap between photos is also recommended. This overlap helps the software to identify the same points in different photos (Hernandez and Lemaire 2017). The object should also be positioned with its longest axis perpendicular to the direction of the camera. Furthermore, internal orientation parameters (IOPs) such as zoom or focus must not differ between shots. However, maintaining consistency of IOPs across an entire dataset can be challenging for both single and multicamera setups (Habib et al. 2014).

\subsection{Post-Processing of the Images}

After image acquisition, blurry or unfocused photos should be discarded, as they usually lead to an inconsistent photo alignment. Before 3D reconstruction, using an image processing software to mask the photos (e.g. Adobe Photoshop) is recommended. In this way, the masked parts of the images such as the background will be excluded from the point cloud generation, thus reducing computational times. These steps will help optimising colour balance of the images, resulting in a more accurate 3D reconstruction and more detailed model texture. 


\section{Conclusions}

This review has highlighted some key features of photogrammetry that make it a useful tool for $3 \mathrm{D}$ reconstruction in biomedical science. It is a non-invasive, low-cost technique that can produce high-resolution surface models. As different setups are possible, it is also a very versatile technique that can be tailored to different applications. However, it is important to be aware of the limitations of the technique and to take steps to obtain high-quality images for accurate 3D reconstruction.

\section{References}

Baghaie A, Tafti AP, Owen HA, et al (2017) Three-dimensional reconstruction of highly complex microscopic samples using scanning electron microscopy and optical flow estimation.

Ball A, Job P, Walker A (2017) SEM-microphotogrammetry, a new take on an old method for generating high-resolution 3D models from SEM images. Journal of Microscopy 267:214-226.

Chandler JH, Buckley S (2016) Structure from motion (SFM) photogrammetry vs terrestrial laser scanning. In: Geoscience Handbook 2016: AGI Data Sheets, 5th edn. American Geosciences Institute, Alexandria, Virginia

Griffith EM, Danilatos GD (1993) Environmental scanning electron microscopy. Wiley-Liss, New York

Estes J, Kline K, Collins E (2001) Remote Sensing. International Encyclopedia of the Social \& Behavioral Sciences: Elsevier Science Ltd: 13144 - 13150.

Eulitz M, Reiss G (2015) 3D reconstruction of SEM images by use of optical photogrammetry software. Journal of Structural Biology 191:190-196.

Evin A, Souter T, Hulme-Beaman A, et al (2016) The use of close-range photogrammetry in zooarchaeology: Creating accurate 3D models of wolf crania to study dog domestication. Journal of Archaeological Science: Reports 9:87-93. 
Ey-Chmielewska H, Chruściel-Nogalska M, Frączak B (2015) Photogrammetry and Its Potential Application in Medical Science on the Basis of Selected Literature. Advances in Clinical and Experimental Medicine 24:737-741.

Faith D, Horsfield CJ, Nazarov W (2006) Characterization of pore size of trimethylol propane triacrylate (TMPTA) polymer foam by pulsed sputter coating and SEM analysis. Journal of Materials Science 41:3973-3977.

Gibelli D, Pucciarelli V, Poppa P, et al (2018) Three-dimensional facial anatomy evaluation: Reliability of laser scanner consecutive scans procedure in comparison with stereophotogrammetry. Journal of Cranio-Maxillofacial Surgery.

Glon F, Flys O, Lööf P-J, Rosén B-G (2014) 3D SEM for surface topography quantification - a case study on dental surfaces.

Habib A, Detchev I, Kwak E (2014) Stability Analysis for a Multi-Camera Photogrammetric System. Sensors 14:15084-15112.

Hermans DJJ, Maal TJJ, Bergé SJ, Catharina J. M. Van Der Vleuten (2013) ThreeDimensional Stereophotogrammetry: A Novel Method in Volumetric Measurement of Infantile Hemangioma. Pediatric Dermatology 31:118-122.

Hernandez A, Lemaire E (2016) A smartphone photogrammetry method for digitizing prosthetic socket interiors. Prosthetics and Orthotics International 41:210-214.

Lane HB (1983) Photogrammetry in Medicine. Photogrammetric Engineering and Remote Sensing 49:1453-1456.

Liu X-J, Li Q-Q, Pang Y-J, et al (2015) Modified method of recording and reproducing natural head position with a multicamera system and a laser level. American Journal of Orthodontics and Dentofacial Orthopedics 147:781-787.

Luhmann T, Robson S, Kyle S (2014) Close Range Photogrammetry: Principles, Techniques and Applications. Whittles Publishing, Dunbeath

Marčiš M (2013) Quality of 3D Models Generated by SFM Technology. Slovak Journal of Civil Engineering 21:13-24. 
Mitchell H, Newton I (2002) Medical photogrammetric measurement: overview and prospects. ISPRS Journal of Photogrammetry and Remote Sensing 56:286-294.

O'Meara SM, Bland JM, Dumville JC, Cullum NA (2012) A systematic review of the performance of instruments designed to measure the dimensions of pressure ulcers. Wound Repair and Regeneration 20:263-276.

Qian S, Sheng Y (2011) A Single Camera Photogrammetry System for Multi-angle Fast Localization of EEG Electrodes. Annals of Biomedical Engineering 39:28442856.

Ripka WL, Ulbricht L, Gewehr PM (2014) Application of a photogrammetric kinematic model for prediction of lung volumes in adolescents: a pilot study. BioMedical Engineering OnLine 13:21.

Ritschl LM, Roth M, Fichter AM, et al (2018) The possibilities of a portable lowbudget three-dimensional stereophotogrammetry system in neonates: a prospective growth analysis and analysis of accuracy. Head \& Face Medicine.

Saad KR, Colombo AS, João SMA (2009) Reliability and Validity of the Photogrammetry for Scoliosis Evaluation: a Cross-Sectional Prospective Study. Journal of Manipulative and Physiological Therapeutics 32:423-430.

Saad KR, Colombo AS, Ribeiro AP, João SMA (2012) Reliability of photogrammetry in the evaluation of the postural aspects of individuals with structural scoliosis. Journal of Bodywork and Movement Therapies 16:210-216.

Skarlatos D, Kiparissi S (2012) Comparison Of Laser Scanning, Photogrammetry And Sfm-Mvs Pipeline Applied In Structures And Artificial Surfaces. ISPRS Annals of Photogrammetry, Remote Sensing and Spatial Information Sciences 1-3:299_ 304.

Stekelenburg CM, Martijn B. A. Van Der Wal, Knol DL, et al (2013) Three-

Dimensional Digital Stereophotogrammetry. Plastic and Reconstructive Surgery 132:204-211.

Stekelenburg CM, van der Wal MBA, Knol DL, et al (2013) Three-Dimensional Digital Stereophotogrammetry. Plastic and Reconstructive Surgery 132:204-211. 
Tafti AP, Kirkpatrick AB, Alavi Z, et al (2015) Recent advances in 3D SEM surface reconstruction. Micron 78:54-66.

Tafti AP, Holz JD, Baghaie A, et al (2016) 3DSEM : Adaptive and intelligent 3D SEM surface reconstruction. Micron 87:33-45.

Ueno A, Osawa H, Suzuki A (1989) Twin-Lens Type Stereoscopic Camera. JP Patent 0117387, 06 Jul 1989

Villa C (2017) Forensic 3D documentation of skin injuries. International Journal of Legal Medicine 131:751-759.

Villa C, Flies MJ, Jacobsen C (2017) Forensic 3D documentation of bodies: Simple and fast procedure for combining CT scanning with external photogrammetry data. Journal of Forensic Radiology and Imaging 10:47-51.

Westoby M, Brasington J, Glasser N, et al (2012) 'Structure-from-Motion' photogrammetry: A low-cost, effective tool for geoscience applications. Geomorphology 179:300-314. 